\title{
Subordination, subordonnées et subordonnants
}

\section{Annie Boone}

\section{(2) OpenEdition}

\section{Journals}

Édition électronique

URL : https://journals.openedition.org/dhfles/2277

DOI : $10.4000 /$ dhfles.2277

ISSN : 2221-4038

Éditeur

Société Internationale pour l'Histoire du Français Langue Étrangère ou Seconde

Édition imprimée

Date de publication : 1 décembre 2002

Pagination : 11-25

ISSN : 0992-7654

\section{Référence électronique}

Annie Boone, «Subordination, subordonnées et subordonnants », Documents pour l'histoire du français langue étrangère ou seconde [En ligne], 29 | 2002, mis en ligne le 01 janvier 2013, consulté le 27 mai 2021. URL : http://journals.openedition.org/dhfles/2277 ; DOI : https://doi.org/10.4000/dhfles.2277

Ce document a été généré automatiquement le 27 mai 2021.

(c) SIHFLES 


\title{
Subordination, subordonnées et subordonnants
}

\author{
Annie Boone
}

\section{Introduction}

1 Dans cette brève introduction à la journée d'étude, les termes subordination, subordonnées et subordonnants sont pris au sens traditionnel : il s'agira de subordination de phrase, de propositions (ou de sous-phrases) subordonnées et de subordonnants de phrases. Dans les grammaires ces termes sont - faut-il le rappeler ? - souvent saisis de façon intuitive et rarement définis de façon rigoureuse. J'évoquerai les principaux problèmes que pose l'utilisation de ces « étiquettes ».

2 La subordination suscite inlassablement l'intérêt des chercheurs (voir les numéros thématiques de revues et les colloques qui lui sont consacrés). La raison est, sans aucun doute, qu'elle focalise un certain nombre de problèmes syntaxiques et sémantiques cruciaux.

\section{Phrase ou proposition?}

3 Faut-il retenir phrase, proposition ou les deux? La proposition est en logique classique un ensemble qui lie un sujet (ce qu'on dit de quelque chose) à un prédicat (ce qu'on en dit) et qui permet l'expression d'un jugement (cf. Arnauld et Lancelot dans La grammaire de Port-Royal, $\left.1676: 28-29^{1}\right)$.

4 Actuellement, la plupart des grammairiens ont renoncé à lier la définition de la proposition à celle du jugement logique et ne retiennent, à la rigueur, que l'aspect grammatical de la définition de Port-Royal (l'union d'un sujet et d'un prédicat). Ici surgit d'ailleurs le problème de la distinction terminologique des énoncés verbaux (les propositions) et des énoncés nominaux (les phrases ${ }^{2}$ ). Ceux qui refusent d'opposer ces deux types d'énoncés utilisent un terme unique, soit celui de proposition ${ }^{3}$, soit celui de phrase ${ }^{4}$. Dès que l'on quitte le terrain de la phrase ou de la proposition simple les 
désaccords disparaissent: les grammairiens ne reconnaissent plus que des "phrases complexes» et admettent en général qu'elles comportent une ou plusieurs «propositions subordonnées».

5 Les critères mélodiques, graphiques et syntaxiques s'avérant souvent impropres à définir la phrase, la linguistique moderne a souvent recours aux notions d'énonciation (l'expressivité de Guillaume : 1919, les voix de Bakhtine : 1929, le modus de Bally : 1932, les embrayeurs [anglais shifters] de Jespersen : 1924, de Jakobson : 1963, etc.) et d'énoncé (le dictum de Bally, le propos de Bonnard : 1981, le message de Grevisse - Goosse : 1986). La phrase pourrait être définie par le biais de l'interaction de l'énonciation et de l'énoncé (cf. Wilmet 1998 : § 558).

6 Les termes principale et subordonnée sont loin aussi de faire l'unanimité. On sait, par exemple, que l'on peut trouver dans la «principale» une information relativement accessoire et dans la «subordonnée » l'essentiel de l'apport informatif ${ }^{5}$. La proposition subordonnée sujet :

1. Que les choses aillent de plus en plus mal est évident

peut aussi poser un problème au grammairien qui n'est guère disposé à voir dans le sujet un complément du verbe. Bref, une proposition subordonnée ne l'est pas toujours au sens propre du mot.

On pourrait, à la suite de Wilmet (1998: § 554), remplacer proposition par phrase, principale par matrice et subordonnée par enchâssée 6 . La phrase simple s'opposerait à la phrase complexe (comportant une phrase matrice et au moins une sous-phrase enchâssée en hypotaxe) et la phrase unique (simple ou complexe) à la phrase multiple (additionnant des phrases simples ou complexes en parataxe). En schéma :

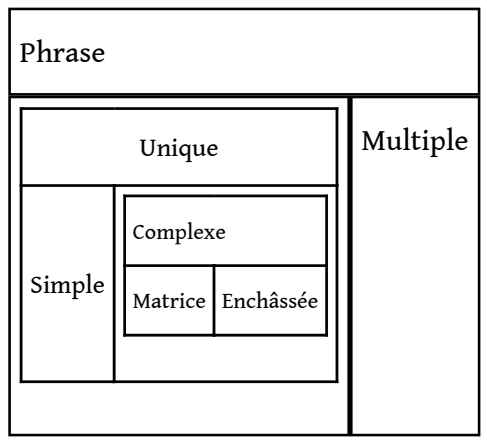

Figure 1 (Wilmet $1998: \S 554)$

\section{Subordonnées}

Les propositions ou phrases subordonnées forment un ensemble hétérogène que les grammaires traditionnelles ne parviennent pas à délimiter de façon rigoureuse. Ainsi, Sandfeld (1965) exclut les propositions infinitives et les participes de son classement des subordonnées, alors que Grevisse (1980) les y intègre. Brunot (1965: 702) reconnaissait déjà avec beaucoup de lucidité que certaines des distinctions proposées ne résistent pas à un examen sérieux et que, par exemple, «il y a des propositions que l'on peut à volonté considérer comme des coordonnées ou des subordonnées ${ }^{7}$. La classe des « subordonnées inversées » : 
2. Tu le lui avouerais qu'il ne te croirait pas (蓲 $\mathrm{Si}$ tu le lui avouais, il ne te croirait même pas) et celle de hiérarchie qu'elle implique sont centrales. Un terme dépend d'un autre, ou lui est subordonné, si son occurrence dans la phrase est nécessairement liée à celle de cet autre, mais non l'inverse : il s'établit ainsi une relation entre un terme régi et un terme régissant ${ }^{11}$. Dans la terminologie de Bloomfield, la subordination est une construction endocentrique, c'est-à-dire une construction dont la distribution est identique à celle de l'un de ses constituants ${ }^{12}$. Depuis la seconde moitié du XIX ${ }^{\mathrm{e}}$ siècle le terme "subordination » est, en grammaire, essentiellement utilisé pour désigner la subordination "propositionnelle ${ }^{13}$. La dépendance qui s'instaure entre la phrase régissante et la phrase dépendante peut, dans certains cas, être décrite comme une relation de rection. Par rapport au verbe, la rection est « un type de dépendance unilatérale où une des constructions est enchâssée dans l'autre de telle sorte qu'elle occupe une place syntaxique d' "argument" par rapport au verbe constructeur » (Deulofeu 1986 : 92). La subordonnée peut alors être reprise par un pronom :

4. Je veux qu'il vienne / Je le veux

5. Qu'il n'ait pas réussi m'étonne / Cela m’étonne comme terme (sujet, objet, circonstant, etc. ...) d'une phrase qui la contient. On constate que les grammaires fondent souvent leur classement des subordonnées sur la fonction qu'elles peuvent remplir dans la phrase ou sur leur possibilité de commutation avec certaines parties du discours (propositions substantives, adjectives, adverbiales). la phrase régissante :

6. Je veux qu'il vienne demain / *Je veux qu'il vient demain 
7. $\mathrm{Il}_{\mathrm{i}}$ veut qu'il ${ }_{\mathrm{ii}}$ vienne / ${ }^{*} \mathrm{Il}_{\mathrm{i}}$ veut qu'il ${ }_{\mathrm{i}}$ vienne / Il veut venir

Les subordonnées ne présentent toutefois pas toutes le même degré de dépendance fonctionnelle. Ainsi, on a relevé l'« indépendance » de certaines circonstancielles, ce qui se traduit, entre autres, par leur permutabilité :

8. Il a réussi malgré qu'il ait été très malade / Malgré qu'il ait été très malade, il a réussi

Pierrard (1994 : 20) a fait remarquer que dans des phrases comme :

9a. Le cocher, qui était ivre, s'assoupit tout à coup

9b. A peine était-il sorti que déjà l'autre arrivait

9c. Il me le demanderait que je ne le lui dirais pas

9d. Plus il mange, plus il a faim

on ne peut plus parler de rection, «puisqu'il n'y a pas de contrainte de dépendance fonctionnelle précise, ni de possibilité de reprise par un pronom ». Il relève néanmoins que divers facteurs attestent que les contraintes de dépendance opèrent toujours (incomplétude syntaxique de chaque proposition prise séparément, contraintes de contrôle sur les temps verbaux et sur les adverbes, contraintes de contrôle de l'antécédent). Les énoncés [9] « reste[nt] encore lié[s] à la notion de dépendance [...] , sous une forme qui rappelle la classique "corrélation” »(Deulofeu 1986 : 90).

Dans certains cas, la dépendance est limitée à l'expression d'un rapport hiérarchique et la deuxième proposition est simplement « greffée » sur la première (op. cit. : 102) :

10. Il faut partir qu'il commence à faire nuit

11. Donne-moi du tabac que je fume (cité par Gadet, 1989 :162)

21 Le morphème que indique simplement que la construction qu'il introduit doit être interprétée par rapport à des éléments du co(n)texte (valeur d'explication, valeur de conséquence,...).

2 La subordination implique une hiérarchisation des énoncés formant une phrase complexe : la phrase matrice - l'intégrante - inclut la phrase enchâssée - l'intégrée - et lui est donc hiérarchiquement supérieure (relation asymétrique, absence de parallélisme entre les deux expressions). On peut concevoir une échelle hiérarchique, qui tiendrait compte des types de contraintes qui existent entre intégrante et intégrée ${ }^{14}$, ainsi que des niveaux d'intégration ${ }^{15}$ (cf. Pierrard 1994 et Melis 1985).

Doit-on concevoir la subordination comme une gradation dans la dépendance (Feuillet 1992), allant d'un pôle supérieur (la parataxe) à un pôle inférieur (les structures enchâssées non finies) ? Deulofeu (1986: 80) s'oppose à cette idée. Il pense «que du point de vue des relations syntaxiques, on peut opposer catégoriellement "rection" et "greffe" " et que "pour le reste, il existe des "effets de gradualité" qui sont dus à l'interférence entre relations syntaxiques et propriétés idiosyncrasiques des subordonnants (valeur lexicale et statut morphologique entre autres) ».

En fait, le processus de la subordination peut être, en syntaxe, appréhendé de deux façons : soit comme un mécanisme d'intégration (combinaison de propositions), soit comme un moyen de translation (transfert catégoriel, cf. Tesnière). Ainsi, pour Guillaume (1973 : 145 sqq.), la complétive par que est le résultat d'une nominalisation propositionnelle ${ }^{16}$, c'est-à-dire d'une opération consistant en la transformation d'une phrase en un nom de discours, un "mot complexe" susceptible d'être utilisé comme élément de la phrase. J'ai montré ailleurs que le mécanisme de nominalisation n'est pas défendable (Boone et Léard 1995 et Boone 1996, 1998b). Ainsi, l'équivalence des propositions complétives et du nom n'est qu'un phénomène accidentel ${ }^{17}:$ le syntagme 
nominal et la que-phrase sont différents en syntaxe et en sémantique ${ }^{18}$. L'explication doit donc être cherchée ailleurs. Je me rallie à la thèse de Léard (1992 et Chevalier Léard 1994, 1996), selon laquelle subordonner, c'est - en particulier dans le cas des complétives - suspendre l'assertion : «[...] la subordination est avant tout, qu'il y ait enchâssement ou non, un problème de modalisation et particulièrement d'évaluation par le locuteur du degré de vérité ou de l'intérêt qu'il accorde à son énoncé » (1994: 47). C'est la thèse que soutiennent également Martin (1983) ${ }^{19}$ et Huot (1991) ${ }^{20}$.

La subordination doit donc recevoir une interprétation sémantique et modale, fondée sur l'analyse syntaxique des phrases matrices et enchâssées. On peut montrer que, à travers la modalisation, la sémantique contrôle le mécanisme syntaxique de la subordination $^{21}$. Lier la subordination à la modalisation permet d'expliquer, entre autres choses, pourquoi nous n'avons pas de complétives derrière les verbes qui expriment intrinsèquement l'aspect ( ${ }^{*}$ Il finit que $P$, ${ }^{*}$ Il commence que $P,{ }^{*}$ Il continue que $P$ ) ou encore derrière des verbes tels que manger ou annuler (*Il mange que $P$, ${ }^{*} I l$ annule que $P)^{22}$. Les faiblesses de l'approche syntaxique, qui voit dans la subordination uniquement un phénomène de dépendance, d'intégration ou encore de nominalisation d'une phrase, sont apparentes ${ }^{23}$.

\section{Subordonnants}

On a adressé à l'appellation " conjonction de subordination » les mêmes critiques qu'à l'étiquette « subordonnée » : qu'est censé relier la phrase enchâssée lorsqu'elle est sujet ?

Les grammaires distinguent généralement entre conjonctions simples et locutions conjonctives. Celles-ci comportent toujours que, ajouté à un autre élément, souvent une préposition, un adverbe ou un nom (avant que, depuis que, malgré que, maintenant que, à la condition que, le temps que, de façon que, etc.). La cohésion interne de ces locutions varie considérablement $^{24}$. Elle peut se traduire par la soudure graphique et/ou des modifications dans la prononciation : il y a, par exemple, soudure sans modification de la prononciation dans quoique et « reviviscence » de la consonne finale de lors [løë] et de puis [p¥i] dans lorsque [løësk \] et puisque [p¥isk \].

28 Les pronoms relatifs, les mots interrogatifs et certains adverbes de degré (qui remplissent une fonction syntaxique dans le cadre de la phrase enchâssée) sont également classés comme subordonnants.

Le Goffic (1993a et b) a proposé un classement nouveau des marqueurs de la subordination qui tient compte de paramètres morphosyntaxiques et sémanticoréférentiels. Il oppose les connecteurs dotés de fonction (type pronominal ou adverbial) et ceux qui ne sont pas dotés de fonction (que complétif). Les connecteurs pronominaux et adverbiaux sont ensuite subdivisés selon leur nature anaphorique ou non anaphorique. Sur cette base Le Goffic définit quatre grands types de subordonnées :

\begin{tabular}{|l|l|l|l|}
\hline TYPE & $\begin{array}{l}\text { CONNECTEUR DOTE DE } \\
\text { FONCTION }\end{array}$ & $\begin{array}{l}\text { CONNECTEUR } \\
\text { ANAPHORIQUE }\end{array}$ & $\begin{array}{l}\text { DENOMINATION } \\
\text { TRADITIONNELLE }\end{array}$ \\
\hline
\end{tabular}




\begin{tabular}{|ll|l|l|l|}
\hline $\begin{array}{l}\text { Percontatives } \\
\text { (pronominales et } \\
\text { adverbiales) }\end{array}$ & + & - & Interrogatives indirectes \\
\hline $\begin{array}{l}\text { Intégratives } \\
\text { (pronominales et } \\
\text { adverbiales) }\end{array}$ & + & - & $\begin{array}{l}\text { Relatives } \\
\text { antécédent, } \\
\text { Circonstancielles en qu- } \\
\text { ou si }\end{array}$ \\
\hline $\begin{array}{l}\text { Relatives } \\
\text { (pronominales } \\
\text { adverbiales) }\end{array}$ & + & & & Relatives avec antécédent \\
\hline \hline Complétives & - & - & Complétives \\
\hline
\end{tabular}

Figure 2

Comme l'a fait remarquer Melis (1994), le classement proposé par Le Goffic a l'avantage de déconnecter les types de subordonnées des fonctions que celles-ci peuvent remplir ${ }^{25}$. Mais il faut remarquer qu'ici aussi la subordination est vue sous l'angle de la nominalisation : Le Goffic (1993a : 80-82 et 1993b : § 22) pose qu'il y a équivalence entre propositions subordonnées et termes simples. Il admet, en particulier, l'équivalence, extrêmement contestable (cf. supra), de la complétive à un groupe nominal.

\section{La subordination dans la grammaire scolaire}

Comme l'a montré Chervel (1977), l'analyse logique du XXe siècle, une fois coupé le cordon ombilical avec le chapsalisme, se préoccupe exclusivement du découpage de la phrase en propositions. Il attribue ce développement d'une nouvelle analyse logique, qui va bouleverser le système des propositions, au fait que "les grammairiens 'découvrent', aux environs de 1870, que les propositions ont les mêmes fonctions dans l'énoncé que les noms et les adjectifs [...]. Toute la laborieuse mise en place du nouveau système, tout l'édifice complexe de ses nouvelles subordonnées, vont résulter de cette simple constatation" (1977: 208). Le terme de proposition subordonnée, qui n'apparaissait que sporadiquement avant 1850 , va s'imposer dans la deuxième grammaire scolaire.

32 La nouvelle terminologie des propositions se met progressivement en place entre 1870 et 1920. On va passer de la dichotomie incidentes 圈 subordonnées, à un système ternaire : relatives菹 complétives 圈 circonstancielles (de temps, de lieu, de manière, de cause). A la liste canonique des sept subordonnées circonstancielles établies vers la fin du XIX siècle (temps, but, cause, conséquence, concession, comparaison et condition) viendra bientôt s'ajouter la proposition participe. L'interrogative indirecte et la proposition infinitive prendront place dans le chapitre des complétives. Après 1920 le nombre des circonstancielles se multiplie : manière, degré, addition, moyen, restriction, etc. Dans des ouvrages récents on voit apparaître une catégorie de subordonnées corrélatives (cf. Grevisse \& Goosse 1993 : § 1075-1077). Dans la plupart des grammaires contemporaines les distinctions sémantiques, qui peuvent être multipliées, continuent à fournir l'ultima 
ratio: cela explique pourquoi le nombre des propositions circonstancielles varie considérablement d'une grammaire à l'autre et pourquoi, par exemple, dans Le Bon Usage (1980: § 2722-2727), les propositions de conséquences ${ }^{26}$ et la "plupart » des propositions de comparaison ${ }^{27}$ deviennent brusquement des propositions qui marquent soit la manière, soit le degré. Le problème réside naturellement dans le fait qu'il s'avère impossible de dresser une liste exhaustive des compléments et des subordonnées circonstanciels ${ }^{28}$. Le chapitre des circonstancielles offre souvent une image fidèle et assez désolante de ce que fut l'ancienne grammaire : les critères sémantiques et formels se bousculent et sont diversement subordonnés l'un à l'autre, des constructions aussi différentes qu'une subordonnée conjonctive, un gérondif et un infinitif prépositionnel sont mises sur le même pied.

Certains grammairiens, renonçant à toute description générale, donnent aux subordonnées le nom de la fonction qu'elles remplissent dans la phrase (subordonnée sujet, attribut, complément d'objet, complément circonstanciel, etc.). D'autres les regroupent d'après la nature du mot qui les introduit ou les assimilent à des parties du discours (subordonnées substantives, adjectives et adverbiales ${ }^{29}$ )

Quand on examine, dans les grammaires, le chapitre qui traite de la subordination, on constate bien souvent que l'utilisation de critères fort divers qui correspondent à des plans d'analyse différents ${ }^{30}$, ne permet pas de délimiter des ensembles exactement superposables et masque bien mal la faillite de la terminologie traditionnelle.

\section{BIBLIOGRAPHIE}

ARNAULD, Antoine - LANCELOT, Claude. [1660] 1676³. Grammaire générale et raisonnée Contenant Les fondemens de l'art de parler expliquez d'une maniere claire \& naturelle ; Les raisons de ce qui est commun à toutes les langues \& des principales differences qui s'y rencontrent; Et plusieurs remarques nouvelles sur la langue Françoise. Édition critique présentée par H. E. BRECKLE. Stuttgart-Bad-Cannstatt : Friedrich Fromann Verlag (fac-similé de la $3^{\mathrm{e}}$ édition).

BAKHTINE, Mikhail. [1929] 1977. Le marxisme et la philosophie du langage. Paris : Éd. de Minuit (traduction française de l'original russe de 1929 par M. Yaguello).

BALLY, Charles. 1932. Linguistique générale et linguistique française. Berne : Francke.

BAT-ZEEV SHYLDKROT, Hava - KUPFERMAN, Lucien (éds). 1995. Tendances récentes en linguistique française et générale. Volume dédié à David Gaatone. John Benjamins : Amsterdam/ Philadelphia.

BILGER, Mireille - VAN DEN EYNDE, Karel - GADET, Françoise (éds). 1998. Analyse linguistique et approches de l'oral. Recueil d'études offert en hommage à Claire Blanche-Benveniste. Leuven : Peeters.

BLANCHE-BENVENISTE, Claire. 1983. «Examen de la notion de subordination ». Recherches sur le français parlé 9. 53-73. 
BONNARD, Henri. 1981. Code du français courant. Paris : Magnard.

BOONE, Annie. 1986. « Réflexions sur le traitement des subordonnées dans "Le Bon Usage" de M. Grevisse ». Travaux de linguistique 12-13. 95-109.

-(éd.). 1994. La subordination. Louvain-la-Neuve : Duculot (Travaux de Linguistique 27).

-1996. « Les complétives et la modalisation ». In : MULLER (éd.). 45-51.

-1998a. «C'est une énigme que cette construction : essai d'analyse d'un "gallicisme” ». In :

ENGLEBERT - PIERRARD - ROSIER - VAN RAEMDONCK(eds). 253-266.

-1998b. « La pronominalisation des complétives objet direct ». In : BILGER - VAN DEN EYNDE GADET (éds). 103-114.

BOONE, Annie - LEARD, Jean-Marcel. 1995. « L'alternance SN / Que P : arguments sémantiques et arguments syntaxiques ». In : BAT-ZEEV SHYLDKROT - KUPFERMAN (éds). 75-94.

BRUNOT, Ferdinand. $1965^{3}$. La pensée et la langue. Paris : Masson et $C^{\text {ie }}$.

CHERVEL, André. 1977. ... et il fallut apprendre à écrire à tous les petits Français. Paris : Payot.

-1983. « Y a-t-il une tradition grammaticale belge ?». Enjeux 4. 73-88.

CHEVALIER, Gisèle - LEARD, Jean-Marcel. 1994. « Le nom subordonnant : les séquences [SN que P] et la modalisation ». In : BOONE (éd.). 43-64.

CHEVALIER, Gisèle - LEARD, Jean-Marcel. 1996. « La subordination nominale : classes, sousclasses, et types sémantiques ». In : MULLER (éd.). 53-65.

CHEVALIER, Jean-Claude - BLANCHE-BENVENISTE, Claire - ARRIVE, Michel - PEYTARD, Jean. 1964. Grammaire Larousse du français contemporain. Paris : Larousse.

CHUQUET, Jean - ROULLAND, Daniel (éds). 1992. Subordination. Rennes : Presses Universitaires.

DAMOURETTE, Jacques - PICHON, Édouard. [1911-1940]. Des mots à la pensée. Essai de grammaire de la langue française. Paris : d'Artrey.

DE POERCK, Guy - BOONE, Annie. 1971. «La syntaxe de la phrase complexe, II. Essai de classification ». Travaux de Linguistique 2. 3-18.

DEULOFEU, José. 1986. «Syntaxe de que en français parlé et le problème de la subordination ». Recherches sur le français parlé 8. 79-104.

ENGLEBERT, Annick - PIERRARD, Michel - ROSIER, Laurence - VAN RAEMDONCK,Dan(éds). 1998. La ligne claire. De la linguistique à la grammaire. Louvain-la-Neuve : Duculot.

FEUILLET, Jack. 1992. « Typologie de la subordination ». In : CHUQUET - ROULLAND (éds). Subordination. 7-28.

GAATONE, David. 1996. « Subordination, subordonnées et subordonnants ». In : MULLER (éd.). 7-13.

GADET, Françoise. 1989. Le français ordinaire. Paris : A. Colin.

GOUGENHEIM, Georges. 1938. Système grammatical de la langue française. Paris : d'Artrey.

GREVISSE, Maurice. $1980^{11}$. Le Bon Usage. Gembloux : Duculot.

GREVISSE, Maurice - GOOSSE, André. $1986^{12}$, 1993³. Le Bon Usage. Paris-Gembloux : Duculot.

GUILLAUME, Gustave. 1919. Le problème de l'article et sa solution dans la langue française. Paris : Hachette. 
GUILLAUME, Gustave. 1973. Leçons de linguistique 1948-1949 C (vol. 3). Québec : Presses de l'Université Laval.

GUIMIER, Claude (éd.). 1993. 1001 circonstants. Caen : Presses Universitaires.

HJELMSLEV, Louis. 1935. « La catégorie des cas ». Acta Jutlandica 7. 1-184.

HUOT, Hélène. 1991. «Quelques aspects syntaxiques de la non-assertion ». In : KREMER (éd.). T. II, 389-401.

JAKOBSON, Roman. 1963. Essais de linguistique générale. Paris : Éd. de Minuit.

JESPERSEN, Otto. 1924. The philosophy of Grammar. Londres : Allen \& Unwin.

KLEIBER, Georges. 1994. «Pronom et anaphore : il dépend-il de son antécédent ? ». In : BOONE (éd.). 167-182.

KLEIBER, Georges - TAMBA, Irène. 1990. « L'hyponymie revisitée : inclusion et hiérarchie ». Langages 98. 7-32.

KREMER, Dieter (éd.). 1991. Actes du XVIIIe Congrès international de linguistique et de philologie romanes. Tübingen : Max Niemeyer.

LEARD, Jean-Marcel. 1992. Les gallicismes. Étude syntaxique et sémantique. Paris-Louvain-laNeuve : Duculot.

LE BIDOIS, Georges - LE BIDOIS, Robert. 1967². Syntaxe du français moderne (2 vol). Paris : A. Picard.

LE GOFFIC, Pierre. 1993a. « Les subordonnées circonstancielles et le classement formel des subordonnées ». In : GUIMIER (éd.). 69-102.

-1993b. Grammaire de la phrase française. Paris : Hachette.

MARTIN, Robert. 1983. Pour une logique du sens. Paris : P.U.F.

MELIS, Ludo. 1985. «Un type particulier de complément circonstanciel : le complément de phrase ». Enjeux 7. 72-85.

-1994. « La typologie des subordonnées circonstancielles et les comparatives ». In : BOONE (éd.). 97-123.

MULLER, Claude (éd.).1996. Dépendance et intégration syntaxique. Tübingen : M. Niemeyer. -1996. La subordination en français. Le schème corrélatif. Paris : A. Colin.

PIERRARD, Michel. 1994. « Subordination, dépendance et hiérarchie : la subordination propositionnelle et ses paramètres d'évaluation ». In : BOONE (éd.). 13-28.

PIOT, Mireille. 1988. « Coordination-subordination. Une définition générale ». Langue française 77. 5-18.

SANDFELD, Kristian. 1965. Syntaxe du français contemporain. II. Les propositions subordonnées. Genève : Droz.

WAGNER, Robert-Léon - PINCHON, Jacqueline. 1962, 1966² $1974^{3}$. Grammaire du français classique et moderne. Paris : Hachette.

WILMET, Marc. $1998^{2}$. Grammaire critique du français. Louvain-la-Neuve : Hachette/Duculot. 


\section{NOTES}

1. «Le jugement que nous faisons des choses, comme quand je dis : la terre est ronde, s'appelle PROPOSITION ; et ainsi toute proposition renferme nécessairement deux termes : l'un appelé sujet, qui est ce dont on affirme, comme terre ; et l'autre appelé attribut, qui est ce qu'on affirme, comme ronde : et de plus la liaison entre ces deux termes, est ». Aristote concevait le jugement logique comme l'union d'un nom au nominatif (o[ nie l'existence dans le temps.

2. Cf. Wagner et Pinchon (1962: § 588) appellent phrases, et non propositions, les énoncés sans verbe à éléments disjoints (Bon, ce café !), les énoncés prédicatifs faits au moyen d'un verbe à l'infinitif (Dormir! ... Rêver peut-être). De même, ils recommandent d'éviter l'emploi de proposition, lorsqu'un élément de la phrase est constitué par un verbe à l'infinitif, au participe ou au gérondif (Il s'est blessé en tombant). Dans la deuxième (1966) et dans la troisième édition (1974), en revanche, la notion de proposition est élargie afin d'y inclure les énoncés comportant un verbe à l'infinitif. Cela illustre bien la confusion terminologique qui peut régner dans les grammaires.

3. Cf. Brunot (1953 : 10-11), G. et R. Le Bidois (1967 : § 1109-1110).

4. Cf. Damourette et Pichon ([1911-1940] : vol. I, § 49), Hjelmslev (1935 : 50-51).

5. Cf. Gougenheim (1938) qui parle de «subordination inverse » lorsque la principale par le sens devient une subordonnée par la forme.

6. Les termes matrice et enchâssée nous ont été légués par la grammaire générative.

7. Cf. Deulofeu (1986:82-83).

8. La solution pourrait être cherchée du côté de l'extension des notions de rection et d'associé (cf. Deulofeu 1986).

9. Cf. Pierrard (1994).

10. A propos des phrases du type C'est une belle invention que le lave-vaisselle et d'un que non subordonnant, cf. Boone (1998a).

11. On notera que la coordination lie des éléments ou des constituants de même nature et de même rang.

12. On aura en revanche une construction exocentrique, si la relation entre les éléments est d'interdépendance, de complémentarité.

13. Le problème de la "subordination référentielle " (cf. Kleiber 1994) ou "sémantique " (cf. Kleiber et Tamba 1990 : 28) ne sera pas abordé ici.

14. De l'absence totale d'indices de rapports hiérarchiques jusqu'à un rapport strictement contraignant.

15. Niveau exophrastique, endophrastique et endosyntagmatique, facteurs liés à la nature du terme régissant ou au type d'opérateur (cf. aussi Boone 1996, 1998b).

16. Guillaume parle ici de nominalisation externe. La nominalisation interne livre une relative (J'ai lu ce livre $\rightarrow$ Ce livre que j'ai lu...).

17. Contrairement à ce que prétendent certains grammairiens et linguistes, on ne trouve, par exemple, pas toujours un $\mathrm{SN}$ dans des positions où l'on peut également avoir une complétive.

18. La catégorie syntaxique $S N$ doit parfois être considérée comme une catégorie sémantique “proposition" sous l'effet du prédicat (ex. Je veux le livre / Je veux que tu me donnes le livre).

19. Selon R. Martin le morphème que « a essentiellement pour fonction de suspendre la valeur de vérité de la proposition qu'il introduit et de la faire dépendre de l'élément verbal ou conjonctionnel qui précède" (1983: 106-107). La fonction sémantique de que est donc fondamentalement une fonction suspensive.

20. Cf. aussi Muller (1996 : 239) : « La subordination peut avoir une justification énonciative [...] : indiquer que le verbe conjugué n'est pas le noyau d'une modalité énonciative, plus spécialement qu'il ne constitue pas une assertion de façon indépendante ». 
21. Il est remarquable que les constructions en que $P$ ne se superposent pas avec les constructions à complément nominal dans les zones où la modalisation est nettement en cause :

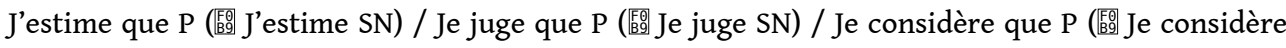
$\mathrm{SN})$.

22. Le fait que l'on n'ait pas *Regarder que $P$, mais bien Voir que $P$ semble lié au fait que regarder est un verbe toujours objectif, alors que voir peut introduire l'idée d'une subjectivité perceptive (comp. aussi Je regarde le ciel bleu à la construction avec attribut de l'objet Je vois le ciel bleu).

23. Cf. Chevalier et Léard (1996) pour une évaluation plus complète de l'approche syntaxique.

24. Cf. Gaatone (1996 : 8-9) : «La cohésion interne de ces séquences varie beaucoup, allant de la plus grande liberté jusqu'à la soudure totale, auquel cas, bien entendu, on n'a plus affaire en réalité à une séquence, mais à un mot unique. Que l'on compare, par exemple, avant que, lequel autorise des insertions diverses, y compris des incises, et lorsque, quoique, bien que, parce que, excluant toute insertion, donc fonctionnant comme un seul mot, que leurs composants soient soudés dans la graphie, ce qui les fait souvent classer comme conjonctions simples, ou non ».

25. Le Goffic (1993a et b) brise l'unité de la locution conjonctive : ce sont des groupes adverbiaux ou prépositionnels composés d'un élément $X$ et incluant une subordonnée que $P$. Un tel « éclatement » avait déjà été suggéré en 1971 par De Poerck et Boone.

26. Déjà traitées $§ 2658$.

27. Déjà traitées $§ 2708$.

28. On peut se demander, par exemple, s'il est légitime de séparer, comme le fait Grevisse (1980) : Il parle en cherchant ses mots (manière) de : C'est en forgeant que l'on devient forgeron (moyen).

29. Cette division que l'on retrouve, entre autres, dans le Bon Usage (1980) et dans la Grammaire Larousse du XXe siècle (1964) et qui est peut-être d'origine allemande, n'a pas eu en France beaucoup d'écho (cf. Chervel 1983).

30. Les relatives et les complétives se définissent à partir du subordonnant (quelle que soit leur fonction), les circonstancielles à partir de leur fonction (quelle que soit leur formation).

\section{RÉSUMÉS}

L'auteur s'interroge d'abord sur la question de l'unité à considérer, appelée tantôt "proposition ", tantôt « phrase » et sur les options terminologiques prises par les grammairiens du français. Pour la notion de subordonnée, l'auteur montre que cette notion est loin d'être délimitée clairement et que la question de la hiérarchie entre les propositions impliquées reste problématique. Quant au transfert de ces notions dans les grammaires pédagogiques, il s'avère particulièrement problématique.

The author wonders at first about the question of the unit to be considered, called sometimes "proposition", sometimes "phrase " and on the terminological options taken by the grammarians of French. As for notion : "subordinate ", the author shows that this notion is far from being clearly bounded and that the question of the hierarchy between the implied proposals remains problematic. As for the transfer of these notions in the educational grammars, it turns out particularly problematic. 
INDEX

Mots-clés : grammaire pédagogique, grammaires du français, phrase, proposition, proposition subordonnée, subordination, terminologie grammaticale

Keywords : educational grammar, grammars of French, grammatical terminology, sentence, subordinate clause, subordination

\section{AUTEUR}

ANNIE BOONE

Vrije Universiteit Brussel 